13,06

\title{
Диэлектрические параметры упруго напряженных, гетероэпитаксиальных пленок $\mathrm{SrTiO}_{3}$
}

\author{
(C) Ю.А. Бойков, В.А. Данилов \\ Физико-технический институт им. А.Ф. Иоффре РАН, \\ Санкт-Петербург, Россия \\ E-mail: yu.boikov@mail.ioffe.ru
}

(Поступила в Редакцию 29 октября 2018 г.)

\begin{abstract}
Методом лазерного испарения на монокристаллических подложках (001) $\mathrm{La}_{0.29} \mathrm{Sr}_{0.71} \mathrm{Al}_{0.65} \mathrm{Ta}_{0.35} \mathrm{O}_{3}$ выращены трехслойные эпитаксиальные гетероструктуры $\mathrm{SrRuO}_{3} / \mathrm{SrTiO}_{3} / \mathrm{SrRuO}_{3}$ с толщиной промежуточного слоя титаната стронция $900 \mathrm{~nm}$. Фотолитография и ионное травление были использованы для формирования на базе выращенных гетероструктур плоскопараллельных пленочных конденсаторов, для которых измерены температурные зависимости тангенса угла диэлектрических потерь при различном напряжении смещения, поданном на электроды из рутената стронция. Визуализированы температурные зависимости диэлектрической проницаемости промежуточного слоя $\mathrm{SrTiO}_{3}$ в сформированных конденсаторных структурах при компенсации внутреннего электрического поля и без нее. Проведен анализ причин резкого увеличения диэлектрических потерь в сформированных пленочных конденсаторах при температурах ниже $50 \mathrm{~K}$.
\end{abstract}

Финансовая поддержка данных исследований была получена из проекта РНФ 16-42-01-067.

DOI: $10.21883 /$ FTT.2019.03.47256.296

\section{1. Введение}

Нелинейные сегнетоэлектрики $\mathrm{Ba}_{1-x} \mathrm{Sr}_{x} \mathrm{TiO}_{3}$ (BSTO) перспективны для применения в перенастраиваемых элементах СВЧ техники (фазовращатели, линии задержки и т. д. [1,2]). Важной для практического использования особенностью элементов на основе BSTO является их работоспособность как при малой, так и при большой мощности высокочастотного сигнала [3]. Отклик диэлектрической проницаемости $(\varepsilon)$ и диэлектрических потерь $(\tan \delta)$ твердого раствора BSTO на электрическое поле зависит от $x$ в химической формуле, от совершенства его структуры, от температуры (Т) и т.д. Использование эпитаксиальных пленок BSTO в комбинации с тонкими слоями (электродами) из высоко проводящих, оксидов (рутенаты, манганиты) позволяет существенно повысить плотность упаковки СВЧ элементов на чипе, понизив при этом величину управляющего напряжения. Применение электродов, выполненных из эпитаксиальных слоев изоморфных по структуре с STO проводящих оксидов, способствует уменьшению концентрации дефектов структуры, как в объеме сегнетоэлектрического слоя так и в области межфазных границ. Зарядовое состояние последних может оказывать существенное влияние на перенастраиваемость гетероструктур, включающих пленки BSTO. Влияние механических напряжений на диэлектрическую проницаемость и на $\tan \delta$ эпитаксиальных пленок BSTO исследовано к настоящему времени лишь фрагментарно.

Титанат стронция $\mathrm{SrTiO}_{3}$ (STO) является начинающим сегнетоэлектриком, спонтанная поляризация в монокристаллах STO отсутствует вплоть до сверхнизких температур [4]. Формирование сегнетоэлектрических доменов в титанате стронция может быть индуцировано механическими напряжениями и/или электрическим полем [5,6]. Рутенат стронция $\left(\mathrm{SrRuO}_{3}\right) \mathrm{SRO}$ изоморфен по структуре с титанатом стронция, его отличительной особенностью является высокая $\left(\sim 10^{22} \mathrm{~cm}^{-3}[7]\right)$ для оксидов концентрация электронов.

\section{2. Эксперимент}

Метод лазерного испарения (ComPex 200, $\mathrm{KrF}, \quad \lambda=248 \mathrm{~nm}, \quad \tau=30 \mathrm{~ns}$ ) был использован для выращивания трехслойных гетероструктур $\mathrm{SrRuO}_{3} /$ $\mathrm{SrTiO}_{3} / \mathrm{SrRuO}_{3} \quad(\mathrm{SRO} / \mathrm{STO} / \mathrm{SRO})$ на подложках (001) $\mathrm{La}_{0.29} \mathrm{Sr}_{0.71} \mathrm{Al}_{0.65} \mathrm{Ta}_{0.35} \mathrm{O}_{3} \quad$ (LSATO). Плотность лазерного излучения на поверхности использованных объемных керамических мишеней SRO и STO составляла $1.5 \mathrm{~J} / \mathrm{cm}^{2}$. Давление кислорода в ростовой камере в процессе формирования трехслойной гетероструктуры $\mathrm{SRO} / \mathrm{STO} / \mathrm{SRO}$ поддерживалось на уровне $0.3 \mathrm{mbar}$, a температура подложки равнялась $760^{\circ} \mathrm{C}$. Толщина промежуточного слоя STO в гетероструктуре SRO/STO/SRO составляла $900 \mathrm{~nm}$, а электроды SRO имели толщину (d) $150 \mathrm{~nm}$. Фотолитография и ионное травление $\left(\mathrm{Ar}^{+}\right.$, $500 \mathrm{~V})$ использовались при формировании верхних электродов $\left(60 \times 60 \mu \mathrm{m}^{2}\right)$ плоско параллельных пленочных конденсаторов и „окон“ для обеспечения контакта с нижним, общим для всех двадцати сформированных на чипе пленочных конденсаторов. Емкость и тангенс угла диэлектрических потерь сформированных конденсаторов измерялись с использованием Agilent 4263b $L C R$ meter. При $T=300 \mathrm{~K}$ разброс в величине емкости и диэлектрических потерь для сформированных на чипе конденсаторов не превышал 20\%. Частота $(f)$ измерительного сигнала равнялась $100 \mathrm{kHz}$, а напряжение 
смещения $\left(V_{b}\right)$ изменялось от -2.5 до $+2.5 \mathrm{~V}$. $V_{b}$ считалось положительным, когда „+““ был подан на верхний электрод. Морфология свободной поверхности выращенных гетероструктур исследовалась с использованием микроскопа атомных сил (режим высоты). Сопротивление $(R)$ верхнего слоя SRO измерялось в геометрии Van der Pauw, а электросопротивление $(\rho)$ рассчитывалось с использованием соотношения $\rho=\pi R d / \ln 2$ [8]. Данные о структуре выращенных гетероструктур были получены с использованием рентгеновской дифракции.

\section{3. Результаты и их обсуждение}

Рентгеновский скан, визуализированный для гетероструктуры SRO/STO/SRO/LSATO, показан на рис. 1. На дифрактограмме, присутствовали только пики (00n) от подложки, электродов и промежуточного слоя STO, что свидетельствует об отсутствии в выращенной гетероструктуре макровключений вторичных кристаллических фаз и о преимущественной ориентации кристаллитов в слоях титаната и рутената стронция относительно нормали к плоскости подложки. Эффективный латеральный размер кристаллитов в выращенных слоях имел величину порядка $200 \mathrm{~nm}$. Изображение свободной поверхности выращенной гетероструктуры показано на вставке $(a)$ на рис. 2. Латеральная разориентация кристаллитов в верхнем электроде SRO имела величину порядка 0.12 градуса (оценка получена с использованием данных о полуширине рентгеновского пика на $\varphi$-скане, визуализированном для рефлекса (111)SRO, см. встав-

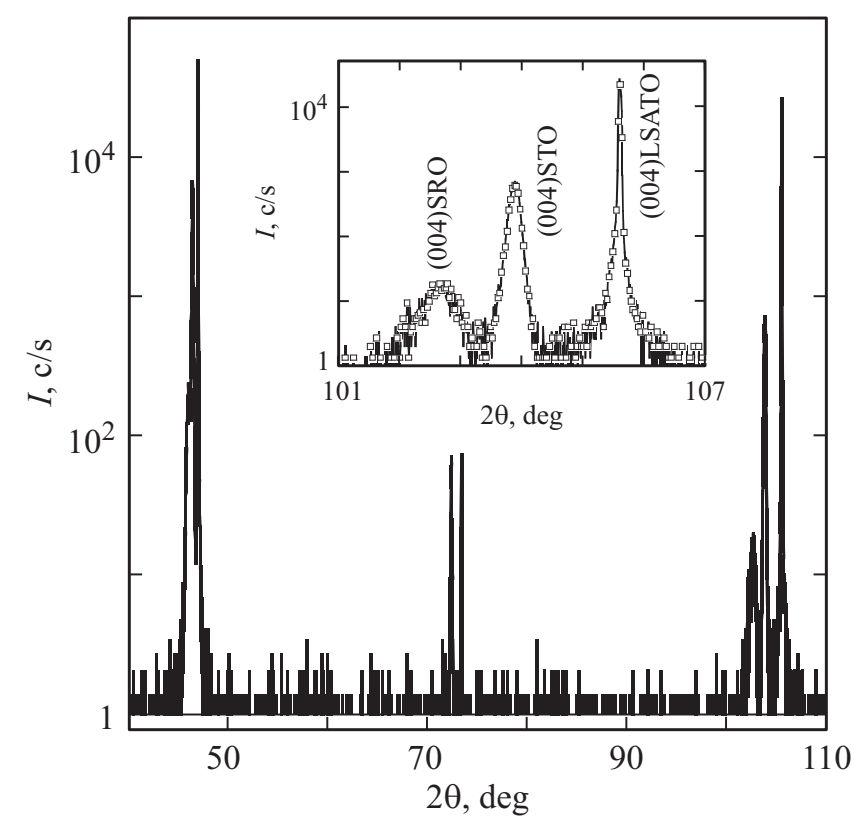

Рис. 1. Рентгеновская дифрактограмма $\left(\omega / 2 \theta, \mathrm{Cu} K_{\alpha 1}\right)$, визуализированная для трехслойной гетероструктуры $\mathrm{SRO} /$ STO/SRO/, сформированной на подложке LSATO. Фрагмент той же дифрактограммы, измеренный при значениях $2 \theta>100$ градусов показан в увеличенном масштабе на вставке.

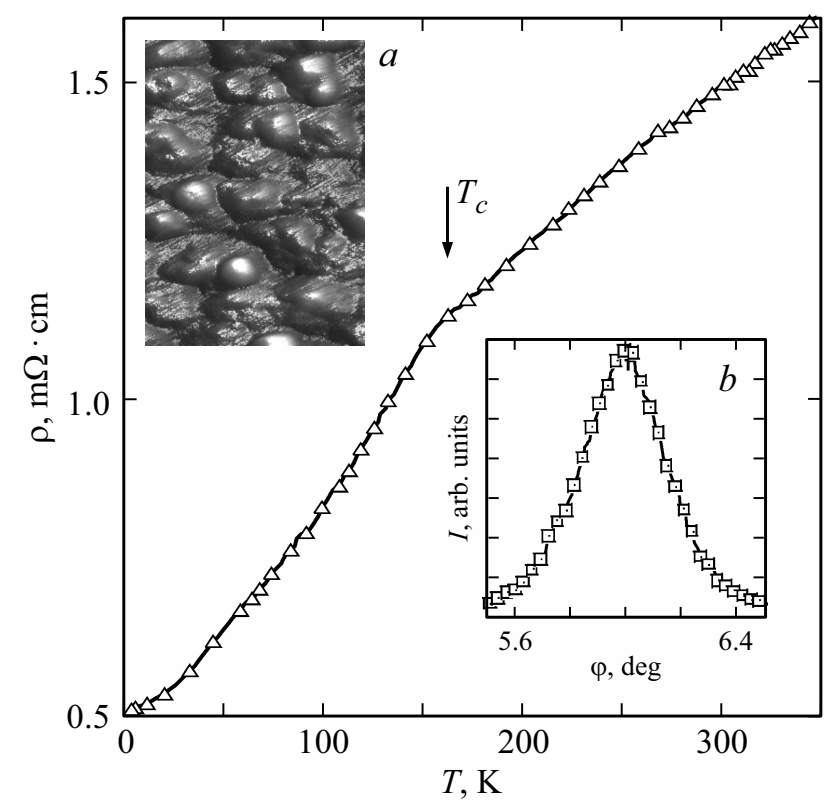

Рис. 2. Температурная зависимость электропроводности верхнего электрода SRO в гетероструктуре SRO/STO/SRO/LSATO. Температура ферромагнитного упорядочения спинов $\left(T_{C}\right)$ в электроде SRO отмечена стрелкой. На вставке $(a)$ показана морфология свободной поверхности выращенной гетероструктуры, на вставке $(b)$ приведен пик на $\varphi$-скане, визуализированном для рентгеновского рефлекса (111)SRO.

ку (b) на рис. 2). Основной причиной азимутальной разориентации кристаллических зерен в выращенных гетероструктурах являлась существенная $(\sim 1.5 \%)$ разница в параметрах кристаллических решеток подложки LSATO и рутената стронция. При $2 \theta>100$ градусов рентгеновские пики от подложки, промежуточного слоя STO и электродов четко разрешались, см. вставку на рис. 1. Пики (004)SRO от верхнего и нижнего электродов в гетероструктуре SRO/STO/SRO в значительной степени перекрывались. Искажение пика (004)SRO со стороны малых значений $2 \theta$ обусловлено тем, что параметр элементарной ячейки нижнего электрода SRO $(3.963 \pm 0.005 \AA)$ вдоль нормали к плоскости подложки был заметно больше соответствующего параметра верхнего электрода $(3.944 \pm 0.005 \AA)$, причем оба были больше параметра псевдокубической элементарной ячейки объемного кристалла рутената стронция $\left(a_{\mathrm{SRO}}=3.927 \AA[9]\right)$. Основной причиной искажения элементарных ячеек в слоях рутената стронция в гетероструктуре SRO/STO/SRO, являются механические напряжения, возникшие вследствие разницы в параметрах кристаллических решеток LSATO, STO и SRO. Из полученных рентгеновских данных следует, что параметр элементарной ячейки промежуточного слоя STO вдоль нормали к плоскости подложки равнялся $3.913 \AA$. Увеличение параметра ячейки слоя STO по сравнению с его значением для стехиометрических объемных монокристаллов титаната стронция $\left(a_{\mathrm{STO}}=3.905 \AA[10]\right)$ 
свидетельствует о двухосном азимутальном сжатии слоя STO в выращенных гетероструктурах. Это хорошо согласуется с данными, представленными в работе [11]. Тип и величина механических напряжений в промежуточном слое STO гетероструктуры SRO/STO/SRO/LSATO зависели от концентрации вакансий кислорода в его объеме, от рассогласования в параметрах кристаллических решеток и температурных коэффициентов линейного расширения материалов, интегрированных в выращенных гетероструктурах.

Температурная зависимость электросопротивления верхнего электрода SRO в выращенной гетероструктуpe показана на рис. 2. $\rho$ уменьшалось с понижением температуры, см. рис. 2. При $T \approx 150 \mathrm{~K}$ наблюдалось существенное изменение наклона кривой $\rho(T)$, обусловленное ферромагнитным упорядочением спинов в слое рутената стронция. Основной причиной азимутальной разориентации кристаллитов в слоях рутената стронция, интегрированных с промежуточным слоем STO в гетероструктуре SRO/STO/SRO/LSATO, является существенное рассогласование в параметрах кристаллических решеток LSATO и SRO.

Температурные зависимости $\tan \delta$ для сформированного пленочного конденсатора, визуализированные при величине напряжения смещения $V_{b}=0,+1$ и $-2 \mathrm{~V}$, показаны на рис. 3. Минимальные значения $\tan \delta\left(\sim 6 \cdot 10^{-3}\right)$ наблюдались при температурах, близких к $70 \mathrm{~K}$. В температурном интервале 70-300 К диэлектрические потери в сформированных пленочных конденсаторах слабо возрастали с температурой, что в значительной степени обусловлено увеличением проводимости слоя STO [12]. Вероятной причиной резкого увеличения $\tan \delta$ с понижением температуры в интервале $50-4.2 \mathrm{~K}$, см. рис. 3 , является формирование в упруго напряженном слое STO сегнетоэлектрических доменов, объемная доля которых в слое STO возрастала с понижением температуры. Появление включений сегнетоэлектрической фазы в механически напряженных образцах титаната стронция наблюдается, как правило, при температурах ниже температуры фазового структурного перехода $(T \approx 110 \mathrm{~K}[13])$. Доменные стенки могут вносить заметный вклад, как в диэлектрическую проницаемость промежуточного сегнетоэлектрического слоя в пленочном конденсаторе, так и в $\tan \delta$ последнего.

На вставке на рис. 3 приведены измеренные при различных температурах полевые зависимости диэлектрической проницаемости промежуточного слоя STO в сформированном пленочном конденсаторе. Внешнее электрическое поле оказывало существенное влияние на диэлектрическую проницаемость промежуточного слоя STO. Следует отметить, что максимум на зависимости $\varepsilon / \varepsilon_{0}$ от напряжения смещения наблюдался при $V_{b} \approx+1 \mathrm{~V}$. Это свидетельствует о присутствии в сформированной емкостной гетероструктуре внутреннего электрического поля $E_{\mathrm{int}} \approx-10 \mathrm{kV} / \mathrm{cm}$, величина которого слабо изменялась с понижением температуры от 50 до $4.2 \mathrm{~K}$. Появление внутреннего поля, направленного

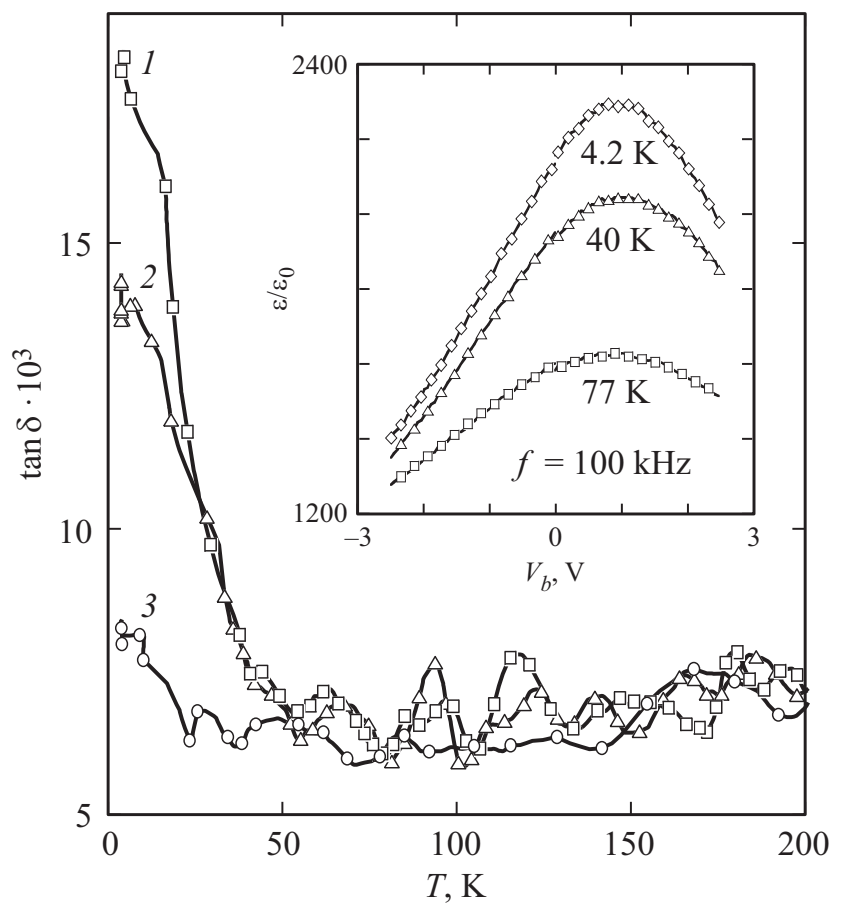

Рис. 3. Температурные зависимости $\tan \delta$ для сформированного пленочного конденсатора, измеренные при различном напряжении смещения, поданном на электроды SRO. $1-V_{b}=+1 \mathrm{~V}, 2-V_{b}=0 \mathrm{~V}, 3-V_{b}=0-2 \mathrm{~V}$. На вставке приведены зависимости $\varepsilon / \varepsilon_{0}$ от $V_{b}$, измеренные при различной температуре (значения $T$ указаны на рисунке).

вдоль нормали к плоскости подложки, обусловлено различиями в зарядовом состоянии верхней и нижней межфазных границ STO/SRO. Существенное влияние на электронные параметры границ оказывают катионные замещения $\mathrm{Ti} \leftrightarrow \mathrm{Ru}$, происходящие в процессе формирования гетероструктуры. Присутствующее в емкостной гетероструктуре внутреннее поле способствовало уменьшению плотности доменных стенок в сегнетоэлектрических доменах, что приводило к уменьшению диэлектрических потерь. Компенсация внутреннего электрического поля при подаче на электроды напряжения смещения $+1 \mathrm{~V}$ сопровождалась существенным увеличением $\tan \delta$, см. рис. 3. При подаче на электроды отрицательного напряжения смещения наблюдалось уменьшение $\tan \delta$.

Визуализированные при $V_{b}=0$ и $V_{b}=+1 \mathrm{~V}$ температурные зависимости диэлектрической проницаемости промежуточного слоя STO в гетероструктуре $\mathrm{SRO} / \mathrm{STO} / \mathrm{SRO}$, показаны на рис. 4. В интервале температуры $70-300 \mathrm{~K}$ зависимости $\varepsilon / \varepsilon_{0}$ от $T$, измеренные при поданном напряжении смещения $+1 \mathrm{~V}$ и без него практически совпадали. Обратная величина диэлектрической проницаемости в указанном температурном интервале линейно возрастала с температурой, см. рис. 4. Из наклона кривой 3 на рис. 4 при $T>100 \mathrm{~K}$ получена оценка постоянной Кюри $\left(C_{0} \approx 0.95 \cdot 10^{5} \mathrm{~K}\right)$ для промежуточного слоя STO в сформированных ге- 


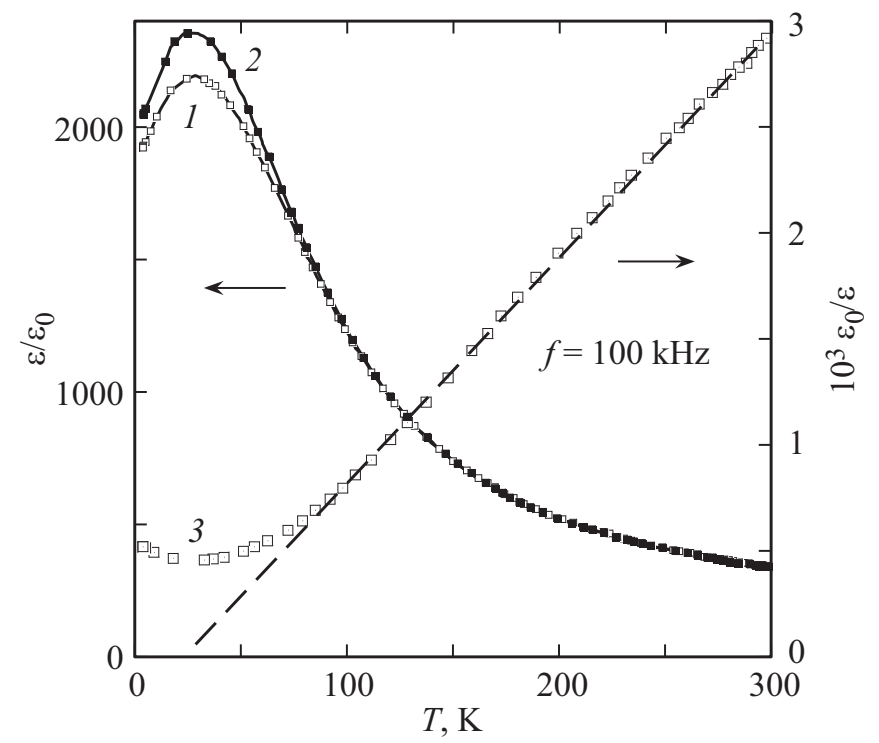

Рис. 4. Температурные зависимости диэлектрической проницаемости промежуточного слоя STO в гетероструктуре SRO/STO/SRO/LSATO, измеренные при $V_{b}=0$ (1) и $V_{b}=+1 \mathrm{~V}(2)$. При $T>70 \mathrm{~K}$ обратная величина диэлектрической проницаемости практически линейно возрастала с температурой, касательная к кривой $\varepsilon_{0} / \varepsilon(T)$ показана на рисунке пунктиром.

тероструктурах. Полученная оценка для $C_{0}$ согласуется соответствующими данными для монокристаллов STO [14]. Появление размытого максимума на температурной зависимости диэлектрической проницаемости, визуализированной для промежуточного слоя STO в выращенных гетероструктурах позволяет получить грубую оценку температуры Кюри $(30-50 \mathrm{~K})$ для включений сегнетоэлектрической фазы, присутствующей в его объеме. На температурной зависимости механически не напряженных объемных кристаллов STO максимум, как правило, не наблюдается [15].

\section{4. Заключение}

Использование лазерной абляции позволяет интегрировать пленки STO с наноразмерными слоями многокомпонентных перовскитоподобных оксидов с высокой проводимостью в эпитаксиальных гетероструктурах. Существенное влияние на диэлектрические параметры пленок STO в гетероструктурах могут оказывать механические напряжения и катионные замещения в области межфазных границ.

\section{Список литературы}

[1] О.Г. Вендик. ФТТ 51, 5, 1441 (2009).

[2] R.A. Chakalov, Z.G. Ivanov, Yu.A. Boikov, P. Larsson, E. Carlsson, S. Gevorgian, T. Claeson. Physica C 308, 3-4, 279 (1998).
[3] О.Г. Вендик, С.П. Зубко. ЖТФ 67, 3, 29 (1997).

[4] K.A. Muller, H. Burkard. Phys. Rev. B 19, 7, 3593 (1979).

[5] J. Hemberger, M. Nicklas, R. Viana, P. Lunkenheimer, A. Loidl, R. Bohmer. J. Phys.: Condens. Matter 8, 25, 4673 (1996).

[6] M.D. Biegalski, E. Vlahos, G. Sheng, Y.L. Li, M. Bernhagen, P. Reiche, R. Uecker, S.K. Streiffer, L.Q. Chen, Gopalan, D.G. Schlom, S. Trolier-McKinstry. Phys. Rev. B 79, 22, 224117 (2009).

[7] P.A. Cox, R.G. Egdell, J.B. Goodenough, A. Hamnett, C.C. Naish. J. Phys. C: Solid State Phys. 16, 32, 6221, (1983).

[8] T.I. Kamins. J. Appl. Phys. 42, 11, 4357 (1971).

[9] J.C. Jiang, W. Tian, X. Pan, Q. Gan, C.B. Eom. Mater. Sci. Eng. B 56, 2-3, 152 (1998).

[10] R.W.G. Wyckoff. In: Crystal Structures. $2^{\text {nd }}$ ed. Interscience, N.Y. 2, 394 (1964).

[11] Luke S.J. Peng, X.X. Xi, B.H. Moeckly, S.P. Alpay. Appl. Phys. Lett. 83, 22, 4592 (2003).

[12] Yu.A. Boikov, T. Claeson. Physica C 336, 3-4, 300 (2000).

[13] J. Dec, W. Kleemann, B. Westwanski. J. Phys.: Condens. Matter. 11, 22, L379 (1999).

[14] A.D. Hilton, B.W. Ricketts. J. Phys. D 29, 5, 1321 (1996).

[15] R.C. Neville, B. Hoeneisen, C.A. Mead. J. Appl. Phys. 43, 5, 2124 (1972).

Редактор Т.Н. Василевская 